\title{
(Sort of) Testing relativity with extreme mass ratio inspirals
}

\author{
Scott A. Hughes \\ Department of Physics and MIT Kavli Institute \\ Massachusetts Institute of Technology, Cambridge, MA 02139
}

\begin{abstract}
The inspirals of "small" $\left(1-100 M_{\odot}\right)$ compact bodies through highly relativistic orbits of massive (several $\times 10^{5} M_{\odot}-$ several $\times 10^{6} M_{\odot}$ ) black holes are among the most anticipated sources for the LISA gravitational-wave antenna. The measurement of these waves is expected to map the spacetime of the larger body with high precision, allowing us to test in detail the hypothesis that black hole candidates are described by the Kerr metric of general relativity. In this article, we will briefly describe how these sources can be used to perform such a test. These proposed measurements are often described as "testing relativity". This description is at best somewhat glib: Because — at least to date - all work related to these measurements assumes general relativity as the theoretical framework in which these tests are performed, the measurements cannot be said to "test relativity" in a fundamental way. More accurately, they test the nature of massive compact bodies within general relativity. A surprising result for such a test could point to deviations from general relativity, and would provide an experimentally motivated direction in which to pursue tests of gravity theories beyond GR.
\end{abstract}

\section{EXTREME MASS RATIO INSPIRALS AND THEIR WAVES}

Compact binary sources in which one member is far more massive than another produce extreme mass ratio inspirals, or EMRIs. Binaries of this sort are expected to form by scattering processes in the nuclei of galaxies: multibody interactions in the dense cluster of stars surrounding a galaxy's central black hole will sometimes place a compact object on an orbit that is so strongly bound to the black hole that it is more likely to inspiral due to gravitational-wave $(\mathrm{GW})$ backreaction than it is to be scattered back out by further interactions. See Clovis Hopman's article in these proceedings [1] for a detailed description.

There has been quite an evolution in our thinking about these sources over the past decade. At the time of the 1st LISA Symposium, it was thought that the inspiralling compact body was most likely to be a white dwarf $[2,3]$; the inferred LISA event rate for white-dwarf-dominated EMRIs was expected to be about an event per year. It is now believed that measured EMRIs will be dominated by the capture of stellar mass $\left(\sim 10 M_{\odot}\right)$ black holes. Though the black hole birth rate is much lower than that of white dwarfs, black holes are captured more efficiently due to mass segregation (the tendency of heavier stellar objects to sink to the bottom of their gravitational potential well) [4]. In addition, black hole EMRIs are "audible" to much greater distances since the EMRI wavestrain scales with the mass of the smaller body. These factors, plus physics such as "resonant relaxation" (see Hopman's article [1] for discussion), have combined to boost 
the estimated event rate to several hundred events per year! Capture might in fact be so efficient that galaxies "eat" all their EMRI sources early in their lives, leaving few low-redshift EMRIs for LISA unless they are replenished through later stellar evolution (a possibility discussed by Sigurdsson and Rees [2]). Conversely, the event rate might be so large that too many EMRIs will be present in LISA data, and the sources may be confused [5]. GW data will have a lot to say about the astrophysics of these sources.

The relativity community has been attracted to this source in large part because it is an amazingly pristine astrophysical system. Since the mass ratio of EMRIs is, by definition, very small $\left(10^{-3} \lesssim m_{1} / m_{2} \lesssim 10^{-7}\right)$, it can be treated as a perturbative parameter. These binaries can thus be analyzed using a variant of perturbation theory, expanding the spacetime (or a suitable surrogate for the spacetime) about the Kerr background of the binary's larger member. Schematically, the binary's evolution can be regarded as a "forced geodesic": Letting $x^{\alpha}$ stand for the coordinates of the smaller body, and writing the connection for the background spacetime as ${ }^{\mathrm{B}} \Gamma_{\beta \gamma}^{\alpha}$, the smaller body follows a trajectory given by

$$
\frac{d^{2} x^{\alpha}}{d \tau^{2}}+{ }^{\mathrm{B}} \Gamma_{\beta \gamma}^{\alpha} \frac{d x^{\beta}}{d \tau} \frac{d x^{\gamma}}{d \tau}=f_{\mathrm{SF}}^{\alpha} .
$$

If the right-hand side were zero, this would simply be the geodesic equation describing motion in the background spacetime. The self force $f_{\mathrm{SF}}^{\alpha}$ that appears instead describes how the motion is changed due to the small body's interaction with its own distortion to the background spacetime. The challenge becomes computing this self force and its impact upon the small body's motion [6,7], and then computing the GWs that this motion generates. See Poisson [8] for a particularly readable review. For our purposes, it suffices to note that computing the self force without any additional simplifying assumptions (other than extreme mass ratio) has proven to be quite a challenge.

Yasushi Mino [9] demonstrated that if the evolution can be regarded as "adiabatic" (in a sense to be quantified momentarily), then the self force can be simplified to something that is relatively tractable:

$$
f_{\mathrm{SF}}^{\alpha}=\frac{1}{2}\left(f_{\mathrm{ret}}^{\alpha}-f_{\mathrm{adv}}^{\alpha}\right) .
$$

(This result is identical in form to the flat space electromagnetic self force originally derived by Dirac [10].) In contrast to the exact expression, which requires integrating a Green's function over the entire past world line of the orbiting body, the "retarded" and "advanced" contributions are relatively simple to evaluate. The assumption of adiabaticity reduces that integration to an averaging procedure; what remains can be used to describe the binary's evolution using tools that are not much more complicated than those which have been developed for simpler cases. See $[11,12,13]$ for further discussion.

The adiabatic assumption that makes it possible to use Mino's "half advanced minus half retarded" prescription amounts to a separation of time scales. We require that the change in any quantity that characterizes the orbit (such as energy or angular momentum) be "small" over a single "orbit". Thus, over short timescales, we can treat the system as following an orbit that neglects radiative backreaction; over long timescales, we treat the inspiral as a "flow" of the system through a sequence of these orbits. 

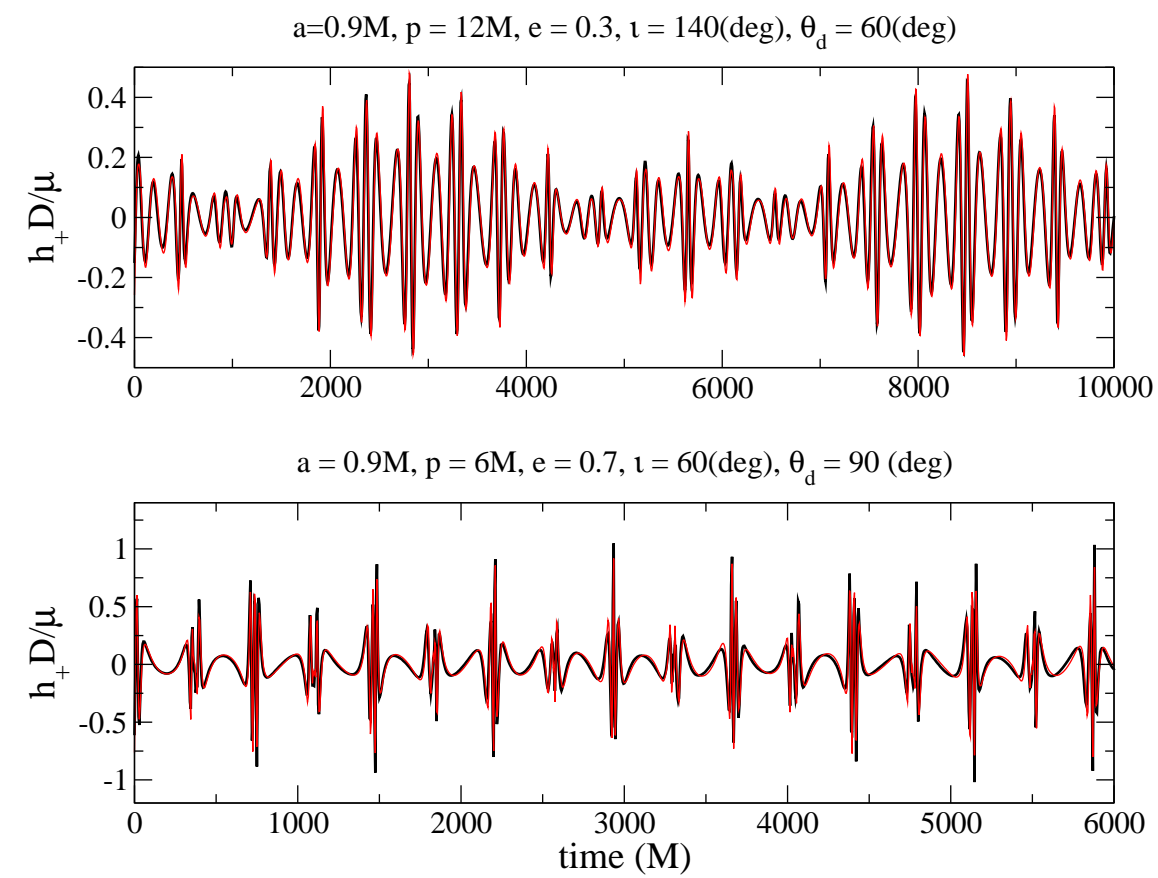

FIGURE 1. Generic EMRI waveforms. Top panel shows waveform generated by a small body in a moderately strong field, moderately eccentric, highly inclined orbit about a rapidly spinning black hole; bottom panel shows waveform for a significantly eccentric and strong field orbit at shallower inclination angle about the same hole. See Ref. [17] for detailed discussion. Superplotted on these waveforms are the "kludge" waves described in Ref. [19] (from which this plot is taken). The kludge matches the strong-field calculation so well that the differences can barely be noted in this plot.

In practice, we currently treat the short timescale orbits as geodesics of the background spacetime. This construction throws away "conservative" components of the self force - a time symmetric, non-dissipative piece of $f_{\mathrm{SF}}^{\alpha}$ that pushes the orbit away from a geodesic of the background spacetime even in the absence of radiative backreaction. Neglect of this piece will lead to a systematic phase error in models of the waveforms [14]; this will have to be fixed to maximize the quality of LISA measurements (though it may be adequate for initial EMRI searches $[15,16])$. Waveforms produced by this approximation are being developed now [17], and appear to be sufficiently accurate for developing and testing LISA EMRI data analysis algorithms. Some examples are shown in Figs. 1 and 2.

It's worth noting that recent work $[18,19]$ has shown that almost all of the features of the waveforms developed in the strong field analysis can be reproduced using a very simple "kludge" approximation in which a flat spacetime wave formula is coupled to the geodesic motion of a small body orbiting a black hole. Indeed, the agreement between the two approaches is somewhat embarrassing - we have superplotted waveforms produced by the "kludge" on the strong field results in Figs. 1 and 2. The differences are very small; in particular, the phase coherency of the two approaches is remarkable. Since the "kludge" waves can be computed many times quicker than the strong-field waveforms (cpu minutes rather than cpu hours), we expect that the "kludge" approach 


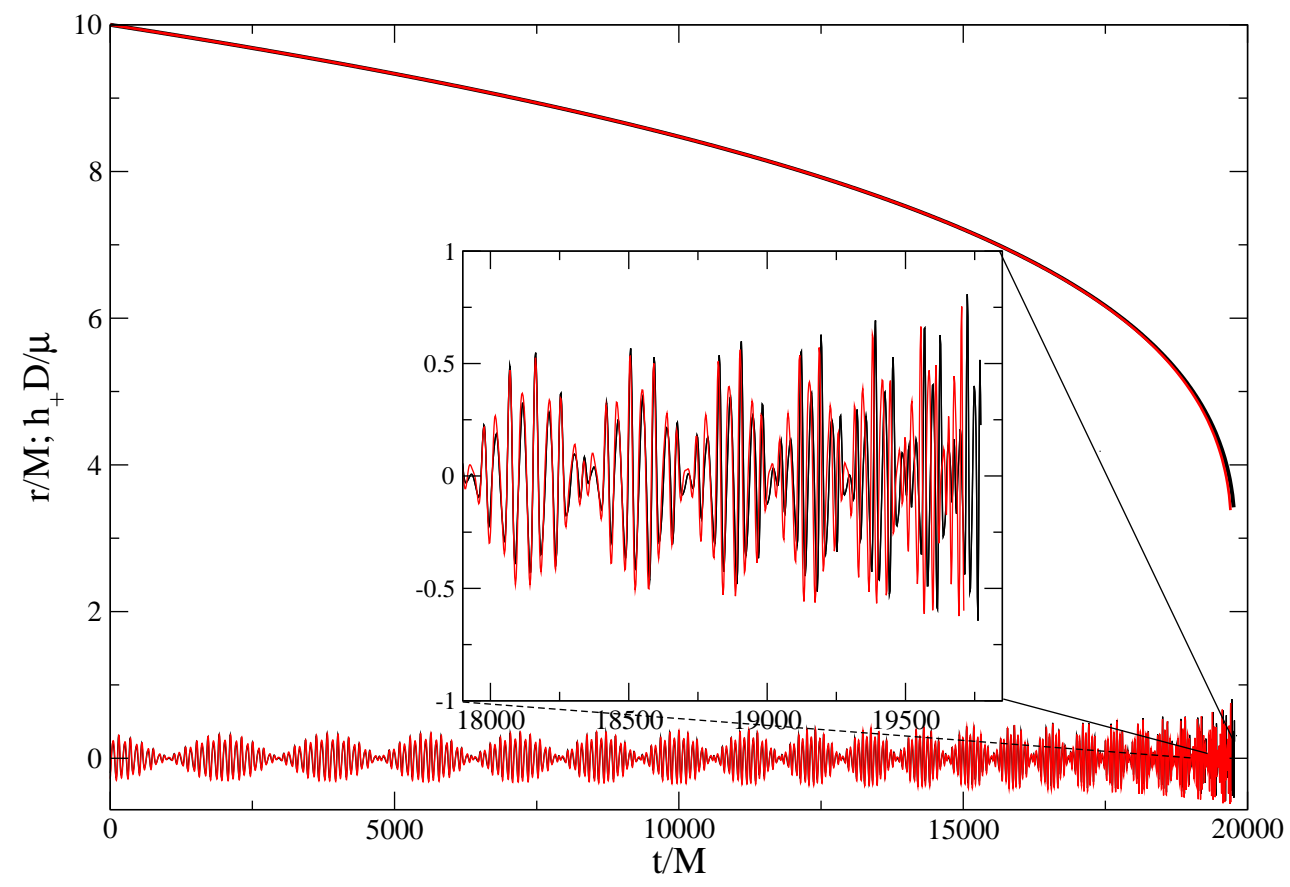

FIGURE 2. Circular EMRI inspiral waveform. This plot shows waves in the (unrealistic) limit of zero eccentricity, but includes the effect of radiative backreaction; the upper curve shows the orbital radius as a function of time, $r(t)$. (The waves shown in Fig. 1 are of sufficiently short duration that the effect of backreaction is neglected.) This example is for inspiral into a rapidly rotating hole with spin parameter $a=0.9$. Superplotted on this waveform is a "kludge" inspiral as described in Ref. [19] (from which this plot is taken). As in Fig. 1, the kludge matches the strong-field embarrassingly well.

will play a key role in scoping out and developing LISA EMRI data analysis.

\section{MAPPING SPACETIMES AND TESTING "BLACK-HOLEY-NESS”}

Comparing the EMRI waveforms that have been developed to the LISA sensitivity shows that they can be tools for high precision studies of black holes. Finn and Thorne [20] showed that, as the small body spirals through the strong field region of the larger black hole, it executes tens to hundreds of thousands of orbits, taking on the order of a year to do so. Because so many orbits accumulate over the measurement, one expects that a fit to a theoretical model will be highly sensitive to small changes to the model's parameters, leading to very accurate parameter determination. Indeed, Barack and Cutler [21] demonstrated using simplified waveform models that LISA should be able to determine many EMRI parameters with exquisite accuracy. In particular, the mass and spin of the larger black hole should be determined with fractional errors of about $10^{-3}-10^{-5}$ (depending upon the detailed nature of the particular EMRI).

Barack and Cutler's analysis assumes that the background spacetime is a Kerr black hole. That such amazing precision is possible under this assumption begs the question of how well we could do if we assume more general spacetimes. In other words, rather 
than just measuring the properties of the assumed black hole, can we test whether the large central object is in fact a black hole, or is something even more exotic?

Fintan Ryan [22] first formulated this question in a way suitable for testing with GW observations. The basic idea is to note that the exterior spacetime of any stationary, axisymmetric body is fully specified by a pair of multipole moment families [23, 24]: a set of mass moments $M_{l}$, and a set of current moments $S_{l}$. These moments label the rate at which certain fields constructed from the metric fall off with distance, as well as their angular dependence. (For a material body, these moments are closely related to the "usual" multipolar distribution of mass and mass motions in the body's interior [25].) As such, the orbits and the GWs generated from the orbits are set by the multipole moments that determine a spacetime's structure. Turning this around, it should be possible to infer the multipole moments which characterize a spacetime by measuring the GWs generated by an object orbiting in that spacetime.

Such a construction is closely analogous to the science of geodesy - inferring the distribution of the earth's mass by studying the orbits of satellites. It is a particularly powerful test for black holes because they have a very special multipole moment structure. Given a Kerr black hole with mass $M$ and spin parameter $a$ (using units in which $G=1=c)$, the moments $M_{l}$ and $S_{l}$ are given by [24]

$$
M_{l}+i S_{l}=M(i a)^{l}
$$

In other words, $M_{0}=M, S_{1}=a M$. All other multipoles are completely determined by these two values, or else are zero.

We thus have a very simple and natural consistency test that the central object must satisfy if it is a Kerr black hole: Measure at least three multipoles. Numbers 0 and 1 fully determine numbers 2 and higher. If any inconsistency with the Kerr relationship (3) is found, then that object cannot be a black hole. This proposed measurement has a similar "moral" foundation to the multimode spectroscopy presented at this meeting by Berti, and discussed by Dreyer et al [26] and by Berti, Cardoso, and Will [27]: the goal is to overdetermine the parameter space to check consistency with Kerr solution.

An initial exploration by Ryan [28] indicates that "black hole geodesy" (which has been named "bothrodesy", based on the Greek root $\beta o \theta \rho o \varsigma$, referring to a sacrificial pit; and "holiodesy", based on the word "hole" and making a pun on "heliodesy") should easily work well enough to test the black hole hypothesis — at least three moments should typically be measured by LISA with better than a few percent accuracy (depending on the large object's mass). As the multipole order is increased, measurement accuracy degrades. Ryan's analysis indicates that as many as 6 or 7 moments may be determined. (Indeed, Ryan's analysis is probably somewhat pessimistic, since for simplicity he only considers circular, equatorial orbits. The additional information encoded by the multiply periodic structure of generic orbits is sure to sharpen our ability to determine a spacetime's geometry.)

Despite this good news, Ryan's analysis can only be considered a proof of principle - as implemented, this spacetime mapping via multipoles is unlikely to work for astrophysical massive objects. In order to make this "assume nothing, measure all multipoles ab initio" scheme work, Ryan must build a spacetime with arbitrary multipole moments. To make a tractable calculation, Ryan essentially abused a weak-field approx- 
imation: He built a spacetime that was strictly accurate only for $r \gg M$ (where $M$ is the mass of the large central object), but used it for all $r$ (including $r \sim$ a few $\times M$ ). In principle one could iterate the algorithm Ryan used to build a spacetime with arbitrary multipoles good for all $r$. In practice, this is unlikely to work well due to the many poorly convergent sums that arise from such an iteration. Effective bothrodesy is likely to require us to set our sights a little lower than an assumption-free measurement of all of a massive body's gravitational multipole moments.

It is often the case that the most effective measurements are of quantities whose values, in some particular framework, should be zero. Motivated by this, we have suggested reformulating bothrodesy as a null experiment. Rather than trying to measure some arbitrary set of multipoles $\left\{M_{l}, S_{l}\right\}$, measure their deviation from the values we would expect if the spacetime were a black hole: $\left\{\delta M_{l}, \delta S_{l}\right\} \equiv\left\{M_{l}-M_{l}^{\mathrm{BH}}, S_{l}-S_{l}^{\mathrm{BH}}\right\}$. We have given the name bumpy black hole to spacetimes in which the multipole deviations $\left\{\delta M_{l}, \delta S_{l}\right\}$ are non zero, since such objects typically have an event horizon, but one that is distorted from that of the Kerr metric [29].

The bumpy black hole is a nice construction since it works very well into the strong field, and also includes the black hole limit in a natural way - simply set the "bumpiness parameters" which control $\left\{\delta M_{l}, \delta S_{l}\right\}$ to zero and we recover the "normal" black hole solutions. Unfortunately, they have a somewhat pathological feature: Bumpy black hole spacetimes contain naked singularities. In retrospect, this is not too surprising: Price's theorem tells us that, in any "normal" situation, a small deviation from a black hole solution should quickly radiate away. Circumventing this mechanism requires some mechanism to prevent normal physics from acting; we are almost guaranteed that these solutions will not be ones that we would encounter in nature. We should bear in mind, though, that the goal of this construction is not to build a spacetime that might exist in nature. Instead, we only want to provide a well-behaved falsifiable straw man with which to test the black hole hypothesis. In this context, it is irrelevant whether deviations from "black holey-ness" come from reasonable physics. (Indeed, we were recently reminded [30] that the idea that a "pure" multipole is not necessarily physical is not unique to gravity. A similar issue applies to electromagnetic multipoles - when we model the electromagnetic field of an object by a sum over multipoles, we don't actually believe that the object contains infinitesimal loops of infinite current, but we accept this fiction for the purpose of computing the object's external fields. The problem is magnified for gravity, however, due to the nonlinearity of the field equations.)

The original presentation of this variant of bothrodesy focused, for simplicity on angular-momentum-free spacetimes (i.e., bumpy Schwarzschild holes) [29]. Since then, Glampedakis and Babak have extended this idea to encorporate spinning black holes [31]. Their "quasi-Kerr" spacetimes amount to Kerr black holes with the "wrong" quadrupole moments. (Though they do not interpret the horizon structure, if any, of these objects, we will for brevity's sake refer to them as "bumpy black holes" since their motivations and structure are sufficiently similar to the original proposal [29].) Though much work remains to compute the GWs that would arise from inspiral in these spacetimes, and also to ascertain whether this construction is ready for mapping the spacetimes of massive compact objects, it is encouraging that the theoretical foundations for this measurement have largely been set. 


\section{IS THIS TESTING RELATIVITY?}

Bumpy black holes assume general relativity in their construction. Thus, strictly speaking, using this framework to map the spacetimes of massive compact objects is not a test of general relativity. It is instead a test of the structure of massive bodies within general relativity.

It is, however, an extraordinarily precise test of that structure. Although work remains to quantify exactly how well these measurements can do, it seems very likely that the null hypothesis - and thus the multipole deviations will be zero, that these bodies

are described by the Kerr solution - can be tested quite rigorously. General relativity provides such a rigid framework that any measured deviation will require explanation. The explanation may turn out to be prosaic - e.g., systematic observation of the "wrong" quadrupole moment may be due to tidal interactions with the black hole's local environment. If not, it may point the way to a new understanding of strong field gravity.

Tather than worrying about whether these measurements are truly testing general relativity or not, we advocate remaining agnostic. General relativity has, to date, been such a formidibly complete and successful theory that it is hard to guess how it might require modification. Rather than looking for wholesale massive deviations in the blueprint, mapping the spacetimes of massive bodies allows us to precisely check the lay of our theory's foundations. In doing so, we also gain a wealth of precise astrophysical information on black hole masses and spins, and on dynamical processes in the cores of galaxies.

\section{ACKNOWLEDGMENTS}

Our work on EMRI science and measurement has benefitted enormously from discussions and collaborations with Stas Babak, Nathan Collins, Steve Drasco, Hua Fang, Éanna Flanagan, Sam Finn, Joel Franklin, Jonathan Gair, Kostas Glampedakis, Daniel Kennefick, Eric Poisson, Saul Teukolsky, and Kip Thorne. Support for this work specifically is provided by NASA Grants NAG5-12906 and NNG05G1056; work on gravitational-wave science generally is supported by NSF Grants PHY-0244424 and PHY-0449884. We also gratefully acknowledge support from MIT's Class of 1956 Career Development Fund.

\section{REFERENCES}

1. C. Hopman, "Astrophysics of extreme mass ratio inspiral sources", contribution to these proceedings; astro-ph/0608460.

2. S. Sigurdsson and M. Rees, Mon. Not. R. Astron. Soc. 284, 318 (1997).

3. S. Sigurdsson, Class. Quantum Grav. 14, 1425 (1997).

4. L. Spitzer, Astrophys. J. 158, L139 (1969).

5. L. Barack and C. Cutler, Phys. Rev. D 70, 122002 (2004).

6. Y. Mino, M. Sasaki, and T. Tanaka, Phys. Rev. D 55, 3457 (1997).

7. T. C. Quinn and R. M. Wald, Phys. Rev. D 56, 3381 (1997).

8. E. Poisson, Living Rev. Relativity 7, 6 (2004); http://www.livingreviews.org/lrr-2004-6. 
9. Y. Mino, Phys. Rev. D 67, 084027 (2003).

10. P. A. M. Dirac, Proc. R. Soc. A 167, 148.

11. S. A. Hughes, S. Drasco, E. E. Flanagan, and J. Franklin, Phys. Rev. Lett. 94, 221101 (2005).

12. S. Drasco, E. E. Flanagan, and S. A. Hughes, Class. Quantum Grav. 22, S801 (2005).

13. N. Sago, T. Tanaka, W. Hikida, and H. Nakano, Prog. Theor. Phys. 114, 509 (2005).

14. A. Pound, E. Poisson, and B. G. Nickel, Phys. Rev. D 72, 124001 (2005).

15. T. Hinderer and E. E. Flanagan, in preparation.

16. M. Favata, in preparation.

17. S. Drasco and S. A. Hughes, Phys. Rev. D 73, 024027 (2006).

18. J. R. Gair and K. Glampedakis, Phys. Rev. D 73, 064037 (2006).

19. S. Babak, H. Fang, J. R. Gair, K. Glampedakis, and S. A. Hughes, Phys. Rev. D, submitted; grqc/0607007.

20. L. S. Finn and K. S. Thorne, Phys. Rev. D 62, 124001 (2000).

21. L. Barack and C. Cutler, Phys. Rev. D 69, 082005 (2004).

22. F. D. Ryan, Phys. Rev. D 52, 5707 (1995).

23. R. Geroch, J. Math. Phys. 11, 2580 (1970).

24. R. O. Hansen, J. Math. Phys. 15, 46 (1974).

25. K. S. Thorne, Rev. Mod. Phys. 52, 299 (1980).

26. O. Dreyer, B. Kelly, B. Krishnan, L. S. Finn, D. Garrison, and R. Lopez-Aleman, Class. Quantum Grav. 21, 787 (2004).

27. E. Berti, V. Cardoso, and C. M. Will, Phys. Rev. D 73, 064030 (2006).

28. F. D. Ryan, Phys. Rev. D 56, 1845 (1997).

29. N. A. Collins and S. A. Hughes, Phys. Rev. D 69, 124022 (2004).

30. S. A. Teukolsky, private communication.

31. K. Glampedakis and S. Babak, Class. Quantum Grav. 23, 4167 (2006). 